\title{
Economic evaluation of ivabradine in the treatment of chronic heart failure in Greece
}

\author{
Georgia Kourlaba ${ }^{1 *}$, John Parissis ${ }^{2}$, Apostolos Karavidas $^{3}$, Alexandra Beletsi ${ }^{4}$, Charalambos Milonas ${ }^{5}$, \\ Neil Branscombe ${ }^{6}$ and Nikos Maniadakis ${ }^{5}$
}

\begin{abstract}
Background: The objective of our study was to assess the cost-effectiveness of ivabradine plus standard care (SoC) in chronic heart failure (CHF) patients with sinus rhythm and a baseline heart rate $\geq 75$ b.p.m. in Greece, in comparison with current SoC alone.

Methods: An existing cost-effectiveness model consisting of two health states, was adapted to the Greek health care setting. All clinical inputs of the model (i.e. mortality rates, hospitalization rates, NYHA class distribution and utility values) were estimated from SHIFT trial data. All costing data used in the model reflects the year 2013 (in €). An incremental cost effectiveness ratio (ICER) per quality-adjusted life year (QALY) gained was calculated. Deterministic and probabilistic sensitivity analyses (PSA) were conducted. The horizon of analysis was over patient life time and both cost and outcomes were discounted at 3.5\% per year. The analysis was conducted from a Greek third party-payer perspective.

Results: The Markov analysis revealed that the discounted quality-adjusted survival was 4.27 and 3.99 QALYs in the ivabradine plus SoC and SoC alone treatment arms, respectively. The cumulative lifetime total cost per patient was $€ 8,665$ and $€ 5,873$, for ivabradine plus SoC and SoC alone, respectively. The ICER for ivabradine plus SoC versus SoC alone was estimated as $€ 9,986$ per QALY gained. The PSA showed that the likelihood of ivabradine plus SoC being cost-effective at a threshold of $€ 36,000 /$ QALY was found to be $95 \%$.
\end{abstract}

Conclusions: Ivabradine plus SoC may be regarded as a cost-effective option for the treatment in CHF patients in Greece.

Keywords: Cost-effectiveness, Heart failure, Ivabradine, Cost-utility

\section{Background}

Heart failure (HF) is a major public health concern worldwide [1]. Almost $1-2 \%$ of the population in European countries suffers from HF, with the prevalence rising to $\geq 10 \%$ among the population aged $\geq 70$ years [2]. Moreover, HF has a poor prognosis as $40 \%$ of patients die within a year from the diagnosis date but thereafter the mortality is less than $10 \%$ per year [3]. Prognosis and patient management are correlated to the severity of heart disease. The main tool used to estimate the heart disease severity is the New York Heart Association (NYHA) classification [4]. Moreover, health-related quality of life (HR-QoL) of HF patients is reduced by the

\footnotetext{
*Correspondence: kurlaba@hua.gr

'The Stavros Niarchos Foundation-Collaborative Center for Clinical Epidemiology and Outcomes Research (CLEO), National and Kapodistrian University of Athens, School of Medicine, Athens, Greece

Full list of author information is available at the end of the article
}

physical, social and emotional limitations imposed by the disease. These symptoms may be caused by the disease itself, by co-morbidities, or can result side effects of treatments [5]. In addition to its substantial effect on morbidity and mortality, HF is one of the most costly chronic diseases in developed countries. Costs associated with HF constitute $1-2 \%$ of all healthcare expenditure [6].

Current treatments aim to relieve and stabilize symptoms and prolong survival by stopping, stabilizing or reversing the progression of HF [7]. Standard pharmacological treatment includes beta-blockers, angiotensin-convertingenzyme (ACE) inhibitors and/or angiotensin receptors blockers (ARBs), aldosterone antagonists and diuretics [5]. Ivabradine (Procoralan ${ }^{\circ}$ ) is a new therapeutic option for patients with chronic heart failure (CHF) in sinus rhythm. Ivabradine is a pure heart rate lowering agent, and represents the first in a new class of agents acting by selective 
and specific inhibition of the cardiac pacemaker $I_{\mathrm{f}}$ current that controls the spontaneous diastolic depolarisation in the sinus node and regulates heart rate. The cardiac effects are specific to the sinus node with no effect on intra-atrial, atrioventricular or intraventricular conduction times, nor on myocardial contractility or ventricular repolarization [8].

The clinical effect of ivabradine plus standard care (SoC) versus placebo on top of $\mathrm{SoC}$ has been evaluated through a Phase III international, multicentre randomised controlled trial (SHIFT trial) [9]. The primary endpoint in SHIFT was a composite of cardiovascular (CV) death or hospitalization for worsening HF. This study showed that ivabradine plus SoC was associated with fewer hospital admissions for worsening HF (first event hazard ratio (HR): 0.74; 95\% Confidence Interval (CI): 0.66-0.83, $\mathrm{p}<0.0001$ ) and lower mortality rates due to HF (HR: 0.74; 95\% CI: 0.58-0.94, p=0.014). Moreover, SHIFT indicated that ivabradine plus SoC was associated with significantly fewer serious adverse events [9].

While the use of ivabradine for the treatment of patients with CHF may be considered to be an effective option, it may impose additional costs to the third party payers and society. The recent climate of the major financial crisis has resulted in strong health care budgetary constraints. This imposes the need to use treatments which are not only clinically effective but also economically efficient, in order to maximize the value for money spent in health care. Simple cross-therapy and cross-country price comparisons are misleading and are not sufficient to determine policies on whether or not (and how) certain treatments should be used. Total treatment cost should be considered and weighed up against the health benefit of a new treatment in relation to existing ones. This need led to the use of economic assessment of technologies used in health care delivery, in the context of which the cost-effectiveness ratio of new treatments is often assessed in comparison to that of existing alternatives. Clinical trials rarely collect sufficient data on treatment costs and consequences for rigorous economic assessment. Thus, mathematical modeling is required to support decision making [10].

Therefore, the purpose of the present study was to conduct an economic evaluation comparing ivabradine plus SoC versus SoC alone in patients with sinus rhythm with NYHA class II-IV CHF and a baseline heart rate $\geq$ 75 b.p.m in Greece.

\section{Methods}

In the present study, an existing Markov model developed for the Health Technology Assessment of ivabradine by National Institute for Health and Care Excellence (NICE), [11] was adapted to the Greek health care setting. The model has been based on the results of the SHIFT trial
[9], taking a third party-payer perspective. Costs and outcomes that occur beyond one year are discounted at a $3.5 \%$ annual rate, consistent with the NICE recommendations [12]. As this study is an economic evaluation analysis and does not involve human subjects no ethics approval issues arise. Input data including human material or human data were derived from other published studies performed with the approval of an appropriate ethics committee.

\section{Model structure}

A simple two state Markov cohort model has been used in the analysis (health states: alive, dead) (Figure 1). Patients enter the model in the "alive" health state and are treated with ivabradine plus $\mathrm{SoC}$ or SoC alone. Then, in each subsequent one month cycle during their life span, patients can either remain alive or die from a CV cause (HF and non-HF CV cause) or non-CV cause.

Alive patients are subdivided into NYHA classes (I, II, III and IV) taking into consideration the SHIFT trial data. The proportion of patients in each NYHA class is modeled to change over time. These NYHA states have been included to capture the quality of life (QoL) implications of HF. The rate of hospitalization was also considered in the model but, as with NYHA class, not as separate health states. The hospitalization rates were indirectly modeled to take into account the associated resource use and transient reductions in QoL. A series of multivariable risk equations have been used to predict the risk of mortality,

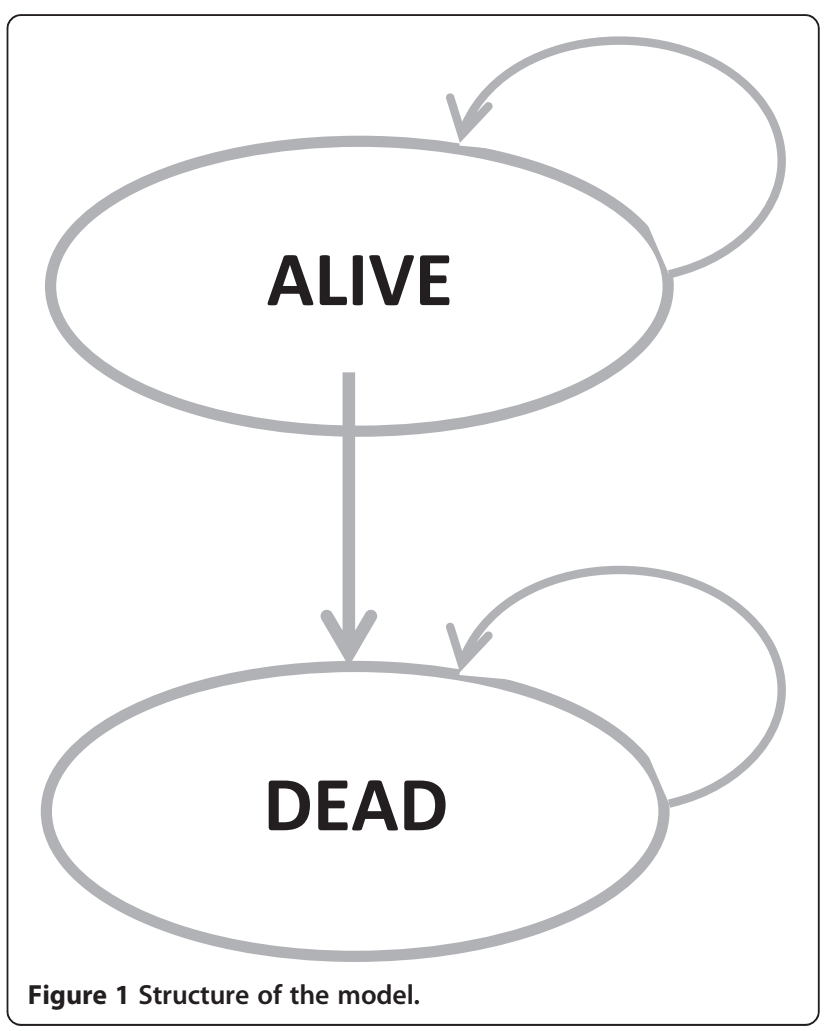


hospitalisation and patient QoL according to treatment allocation and a range of patient baseline characteristics selected on the basis of the SHIFT study protocol, a previous HF risk equation and clinical advice, as described in detail below.

The key assumptions considered in the model are briefly presented in Table 1 and described below in detail.

\section{Comparators/schedules}

In the present analysis, ivabradine plus SoC therapy is compared to $\mathrm{SoC}$ alone. SoC is considered equivalent across treatment groups. In particular, the SoC drugs included in the analysis reflect those drugs currently recommended in ESC guidelines (beta-blockers, ACE inhibitor, diuretics, aldosterone antagonists, ARBs, cardiac glycosides).

\section{Model inputs}

\section{Mortality data}

In the base case analysis, $\mathrm{CV}$ and non-CV mortality was considered separately. The risk of CV mortality in patients who do not receive ivabradine was estimated based on SHIFT SoC data, by using multivariable risk equations for both the within-trial (29 months) and post-trial

\section{Table 1 Summary of key assumptions considered in the} cost-effectiveness model

\begin{tabular}{|c|c|}
\hline Parameter & Key assumptions \\
\hline Model structure & Two-stage Markov cohort model \\
\hline CE model time horizon & Lifetime \\
\hline Model cycle & Monthly \\
\hline CV mortality for SoC & $\begin{array}{l}\text { SHIFT data by applying Gompertz } \\
\text { survival model }\end{array}$ \\
\hline $\begin{array}{l}\text { HR for CV mortality of } \\
\text { ivabradine vs SoC }\end{array}$ & 0.90 (0.80 - 1.03) based on SHIFT data \\
\hline Rate hospitalization & SHIFT data by applying Poisson model \\
\hline $\begin{array}{l}\text { Rate ratio for hospitalization } \\
\text { of ivabradine vs SoC }\end{array}$ & $0.83(0.78-0.93)$ based on SHIFT data \\
\hline Length of hospitalization & Local data (based on experts' opinion) \\
\hline Utility data & $\begin{array}{l}\text { SHIFT data by applying mixed } \\
\text { regression model }\end{array}$ \\
\hline NYHA data & $\begin{array}{l}\text { SHIFT data by applying adjusted } \\
\text { ordered logistic regression }\end{array}$ \\
\hline Ivabradine treatment effect & Cardiovascular endpoint \\
\hline Ivabradine use (years) & Lifelong \\
\hline Cost discount rate per annum & $3.5 \%$ \\
\hline Effects discount rate per annum & $3.5 \%$ \\
\hline $\begin{array}{l}\text { Resource utilization and } \\
\text { unit costs }\end{array}$ & $\begin{array}{l}\text { Local data (government gazette and } \\
\text { experts' opinion) }\end{array}$ \\
\hline Outcome mesaures & $\begin{array}{l}\text { QALYS, LYS, ICER per QALY and per LY } \\
\text { gained, lifetime total cost }\end{array}$ \\
\hline
\end{tabular}

CE: cost-effectiveness; HR: hazard ratio; QALYs: quality-adjusted life years; LYs: life years; ICER: incremental cost effectiveness ratio; CV: cardiovascular; NYHA: New York Heart Association. extrapolated period. In the base case analysis, the CV mortality risk for patients who received SoC alone was estimated by applying a parametric survival regression model on the mortality data of the full SHIFT cohort. The Gompertz distribution was found to best fit the observed mortality data based on statistical evidence (AIC and BIC criteria), a visual review of Kaplan-Meier survival plots versus predicted curves and the plausibility of predicted survival in the extrapolated, post-trial period [11]. Survival models based on the exponential and Weibull parametric distributions, the next best fitting distributions, and Kaplan Meier data, were included in the model as part of the sensitivity analysis [11].

The CV mortality risk equation was estimated adjusting for a series of baseline patient characteristics. The purpose of including these covariates was to generate different estimates of mortality, depending on the baseline characteristics of populations. The covariates considered for the analysis were patient baseline characteristics; baseline use of heart failure medications; prior use of other cardiac therapies; medical history: prior event; Patient biological characteristics.

The treatment effect on CV mortality for ivabradine plus $\mathrm{SoC}$ was estimated by applying a relevant treatment effect (HR) obtained from the parametric model to the underlying mortality risk associated with the $\mathrm{SoC}$ group. Specifically, the HR of 0.90 obtained from the Gompertz model was applied.

A separate sensitivity analysis considering only the cause-specific HF mortality endpoint was also considered (with non-HF CV death modeled equivalent to SoC) [11].

The non-CV mortality rate was modeled to be equivalent for ivabradine plus $\mathrm{SoC}$ and $\mathrm{SoC}$ alone. This rate was estimated by using the age and sex adjusted Greek mortality rates based on the latest data of National Statistics Service (www.statistics.gr).

\section{NYHA class}

As mentioned above, the proportion of patients in each NYHA class was considered in order to capture the QoL of HF patients. In the base case analysis, the distribution of patients in each NYHA class was estimated by applying a generalized ordered logistic regression model (a proportional odds model) on the SHIFT data. The regression model was designed to predict a time adjusted NYHA distribution for each treatment arm, separately. Because of the lack of any evidence to predict the distribution of patients by NYHA class beyond the SHIFT trial period, we assumed that the proportions remained fixed after the trial period. This proportion was set equal to that observed in the trial at 29 months (LoCF). This approach was considered more conservative than the extrapolation of SHIFT data using the ordered logistic regression [11]. 


\section{Hospitalizations}

As with the NYHA class, the hospitalization rates related to HF, other CV causes and all causes for both arms under study were considered in the model to capture the appropriate resource use and the impact on patient's QoL. These rates were estimated through Poisson regression models applied on the SHIFT data. In the base case analysis, the all-cause hospital admissions were considered. The treatment effect of ivabradine on the rate of admissions to hospital was estimated using a rate ratio of 0.83 derived from the Poisson regression model. The treatment effect was modeled on the all-cause admission because $\mathrm{CV}$ and HF admissions were assumed to be implicitly captured in all-cause admission. Admission to hospital after the trial was modeled to be equivalent to the within-trial period and assumed to occur at a constant rate throughout the model irrespective of the ageing population [11].

\section{Hospitalization length of stay and type of ward}

The length of stay (LoS) associated with hospital admission was estimated using external data based on experts clinical advice. The average LoS was varied according to diagnosis on hospital admission (i.e. HF, other CV diagnosis and non-CV diagnosis). In the base case model, an average LoS of 5 days for all-cause hospitalizations were applied according to data obtained from ministry of health. The average LoS for HF and other CV diagnosis was set at 7 and 4 days, respectively, based on local expert's advice. Moreover, the experts reported that only $30 \%$ of patients with HF spend 3 days in intensive care units (ICUs) and the remaining 4 days in cardiac units, while $70 \%$ of patients are hospitalized only in cardiac units.

\section{Utilities}

Utility values describe the health-related QoL associated with different health states on a scale of zero to one, where zero is equivalent to death and one represents best imaginable health. Due to lack of local utility values for patients with HF, the utility values taken from SHIFT-PRO study in which health-related QoL was captured with EQ$5 \mathrm{D}$ questionnaire were considered in the model (Table 2). EQ-5D scores were derived using UK population tariff values [13]. A mixed regression model (designed for longitudinal datasets with repeated observations within respondents) was used to analyze SHIFT EQ-5D scores. The clinical variables considered to potentially predict patient QoL were consistent with those considered in the CV and hospitalization risk equations plus two additional timevarying variables - hospitalization within a 60 day time interval (EQ-5D visit date \pm 30 days) and NYHA class [11].

\section{Costing methods}

The present economic evaluation was conducted by the third-party-payer perspective and as such only health
Table 2 Utility values and costing data considered in the model

\begin{tabular}{|c|c|c|}
\hline Parameters & Values & Source \\
\hline \multicolumn{3}{|l|}{ Utility values } \\
\hline No hospitalization & & SHIFT trial \\
\hline NYHA I & 0.82 & \\
\hline NYHA II & 0.74 & \\
\hline NYHA III & 0.64 & \\
\hline NYHA IV & 0.46 & \\
\hline Hospitalization & & SHIFT trial \\
\hline NYHA I & -0.04 & \\
\hline NYHA II & -0.07 & \\
\hline NYHA III & -0.10 & \\
\hline NYHA IV & -0.29 & \\
\hline \multicolumn{2}{|l|}{ Drug costs per month } & price bulletin issued by the Greek \\
\hline Standard care & $€ 35.26$ & $\begin{array}{l}\text { Ministry of Health [15], law } \\
\text { 4052/2012, Government Gazette }\end{array}$ \\
\hline Ivabradine & $€ 40.52$ & \\
\hline \multicolumn{3}{|l|}{$\begin{array}{l}\text { Other therapy related } \\
\text { costs }\end{array}$} \\
\hline ECG & $€ 3.44$ & \\
\hline $\begin{array}{l}\text { Hospitalization cost } \\
\text { (cost per diem) }\end{array}$ & & $\begin{array}{l}\text { Government Gazzette, Ministerial } \\
\text { Desicion 104494, 26/9/2011 }\end{array}$ \\
\hline Intensive Care Unit & $€ 200$ & \\
\hline Cardiac Units & $€ 110$ & \\
\hline Other resource use & & $\begin{array}{l}\text { Government Gazzette [16] and } \\
\text { local experts' opinion }\end{array}$ \\
\hline $\begin{array}{l}\text { On-going HF } \\
\text { management costs }\end{array}$ & $€ 26.05$ & \\
\hline
\end{tabular}

HF: Heart Failure; ECG: electrocardiographic changes; NYHA: New York Heart Association.

care costs reimbursed by the payer were considered in the analysis. Any other cost, such as the costs related to the central Government budget to cover personnel salaries or patients copayments, was not considered. In particular, hospitalization, medication and HF management costs were considered in the model. All costs (in $€$ ) reflect the year 2013.

To estimate the total hospitalization cost, the cost per day for each ward type (i.e. intensive care unit (ICU), general medical, cardiac and rehabilitation) was multiplied by the LoS and the hospitalization rate (described above). The LoS in each ward type as well as the probability of being hospitalized to a cardiac unit or ICU was obtained from local experts. Reimbursement unit costs for bed-day were obtained from the Diagnostic Related Groups (DRGs) tariffs issued by the Greek Ministry of Health [14].

The total monthly cost of ivabradine has been estimated by considering the proportion of patients using $2.5 \mathrm{mg}$ (10\%), $5 \mathrm{mg}$ (70\%) and $7.5 \mathrm{mg}$ (20\%) in Greece (based on experts advice) and the current price of ivabradine as it was obtained from the latest (February 2013) price bulletin 
issued by the Greek Ministry of Health [15] (Table 2). The price of $2.5 \mathrm{mg}$ was assumed to reflect a half dose of the $5 \mathrm{mg}$ cost, and it is consistent with clinical practice (i.e. the scored tablets may be halved).

The total monthly cost of SoC has been modeled using the overall proportions of patients using each SoC therapy based on local experts opinion, the mean daily dose for each drug as it was reported by local experts and the relevant drug prices (calculated as the cost per $\mathrm{mg}$ ) (Additional file 1, Table 2). Note that the proportions and daily doses reported by the local experts where in line with those taken by patients in SHIFT study. In case that more than one drug was reported from the experts for a particular SoC therapy group, the weighted cost was considered in the model (Additional file 2). All drug prices were obtained from the latest (February 2013) price bulletin issued by the Greek Ministry of Health [15].

The analysis was undertaken from a payer perspective, hence drug cost was based on the retail product price minus the patient copayment (i.e. reimbursed cost). Moreover, cost is net of two different rebates paid by manufacturers (law 4052/2012, Government Gazette). In particular, a rebate of $9 \%$ on the ex factory price is applied as a return for products to be included into the positive list (i.e. a list of reimbursed products). Additionally, a volume rebate, ranging from 0 to $8 \%$, is also applied on the ex factory price based on the quarterly sales of each product. In the present analysis a $5 \%$ is assumed based on a realistic assumption about possible sales.

An additional one-off cost was also included for ivabradine therapy titration. Based on local experts advice, dosage titration, in Greece, takes place in a routine visit at the outpatient department of hospital as soon as an ECG has been undertaken. However, social security funds do not reimburse the outpatient visit and as a resultthe dosage titration cost for ivabradine group was considered to be equal to the cost of ECG as it was obtained from the Government Gazette (FEK B' 3054/2012).

The other modeled resource use for SoC included the on-going HF management (physician visits, outpatient procedures, tests). The number of visits, the laboratory tests (blood and biochemical tests) and cardiological tests (e.g. echo, MRI etc.) required were retrieved from local experts. The health care utilization and costs for managing HF were obtained from Government Gazette [16] and local experts (Table 2).

\section{Data analysis}

The base case approach and data were used to get mean estimates of life time costs, life years (LYs) and qualityadjusted life years (QALYs) for each comparator. When new options like ivabradine are more effective (i.e. higher QALY) and less costly than comparators, they are considered as "dominant" treatments. In cases where new options are less effective and more costly they are considered as "dominated" by the alternatives. In cases where they are associated with higher QALY and higher cost they are considered as cost-effective only when the incremental cost-effectiveness ratio (ICER) is lower than a specific predetermined threshold [17-19]. For a treatment to be considered cost-effective a threshold of $€ 36,000$ per QALY was used in the current analysis. This is based on the WHO guidelines that states that a treatment should be considered cost-effective if the ICER is between 1 or 3 times the gross domestic product (GDP) per capita of that country and a treatment is considered highly cost effective at less than 1 times the GDP per capita [20]. The GDP per capita in Greece was estimated at $€ 18,000$ taken from the IMF estimation of GDP per capita using current prices [21].

The majority of input data used in the current model are subjected to variation. Therefore, a probabilistic sensitivity analysis (PSA) was conducted to quantify uncertainty. Multivariate regression functions generated using SHIFT individual patient data have been entered in the model along with a Cholesky decomposition to account for correlated parameters. Monte Carlo simulation has been used to generate the resulting joint distributions of total costs and QALYs in the model [22]. The model outputs have also been expressed in terms of 'decision uncertainty' using cost-effectiveness acceptability curves (CEACs) which show the probability of each therapy being cost-effective given a particular threshold value for cost-effectiveness [23].

Deterministic sensitivity analysis was performed to identify the parameters and structural assumptions to which the model was most sensitive. The upper and lower bounds of parameters were defined by $95 \%$ CIs where possible or plausible variation around the base case values.

Subgroup analyses have been performed for subgroup populations identified from the clinical study protocol. These included age ( $<$ or $\geq 75$ years old); HF duration (categorized by quartile cut points); NYHA class; Left Ventricular Ejection Fraction (categorized by quartile cut points); prior ischaemia; prior diabetes and $\beta$-blocker use.

All statistical calculations performed using Microsoft Excel 2010.

\section{Results}

\section{Deterministic results}

In the base case analysis, the Markov model revealed that the discounted survival was higher in ivabradine plus SoC arm compared to the SoC alone by $0.25 \mathrm{LYs}$, while the corresponding discounted QALYs were increased by 0.28 QALYs.

Moreover, it was found that ivabradine plus $\mathrm{SoC}$ was the most costly treatment regimen versus SoC alone, in the lifetime horizon ( $€ 8,665$ versus $€ 5,873$, respectively). 
Among the cost categories considered, drug acquisition costs account for $61 \%$ and $40 \%$ of total costs in ivabradine plus SoC and SoC alone, respectively. Following drug acquisition cost, hospitalization cost accounts for $21 \%$ and $30 \%$ of the total costs of ivabradine and SoC, respectively. Finally, follow up costs made up approximately $30 \%$ of the total cost for SoC and $17 \%$ for ivabradine. Under the base case assumptions, incremental analysis showed that ivabradine plus SoC was more effective and more costly than SoC alone, resulting in an ICER equal to $€ 9,986$ per QALY gained indicating that ivabradine plus SoC was a cost-effective alternative (Table 3).

\section{One way sensitivity analysis}

\section{Parameter sensitivity analysis}

The results of the sensitivity analysis conducted on parameter estimates are reported in the Tornado diagram featured in Figure 2. It should be noted that the Tornado diagram has been estimated by applying average covariate values into the risk equations for simplicity (base case ICER estimate derived using this method €9,196 - approximately $€ 790$ less than the base case ICER estimate $€ 9,986$ - which was derived by applying individual patient profiles into the risk equations one at a time). The results of sensitivity analysis indicated that the ICER was likely to remain below the threshold of $€ 36,000$ per QALY gained in all scenarios, with the exception of the ivabradine HR for CV mortality where the ICER exceeded $€ 36,000$ when the HR was set at the upper limit of its $95 \%$ confidence interval. The rest of findings of this analysis suggested a robustness of results to clinically plausible changes in assumptions.

Table 3 Deterministic cost - effectiveness results of Base Case Lifetime Analysis

\begin{tabular}{llll}
\hline & $\begin{array}{l}\text { Ivabradine + Standard } \\
\text { therapy }\end{array}$ & $\begin{array}{l}\text { Standard } \\
\text { therapy }\end{array}$ & Incremental \\
\hline Costs & & 5,873 & 2,792 \\
Total costs $(€)$ & 8,655 & 2,374 & 2,966 \\
$\begin{array}{l}\text { Drug acquisition } \\
\text { cost }(€)\end{array}$ & 5,340 & & \\
Hospitalization & 1,833 & 1,781 & 52 \\
costs $(€)$ & & 1,754 & -262 \\
HF management & 1,492 & & \\
costs $(€)$ & & 3.99 & 0.28 \\
Health outcomes & & 5.61 & 0.25 \\
QALYs & 4.27 & & \\
LYs & 5.86 & & 9,986 \\
Incremental & & & 11,002 \\
analysis & & & \\
ICER per QALY $(€)$ & & & \\
ICER per $L Y(€)$ & & & \\
\hline
\end{tabular}

ICER: incremental-cost-effectiveness ratio; LY: life-year; QALY: quality-adjustedlife-year.

\section{Structural sensitivity analysis}

The results of the structural sensitivity analysis are reported in Table 4. The structural scenario analyses indicate that assumptions have generally been conservative with respect to the ICER. The use of alternative parametric distributions (exponential and Weibull) for CV mortality estimation also both resulted in a more favorable ICER estimate, while the use of Kaplan Meier data of SHIFT study to estimate CV mortality resulted in a higher ICER still below the threshold of $€ 36,000$ per QALY gained. Moreover, structural sensitivity analysis showed that when the treatment effect of ivabradine was modeled only on HF mortality, with other CV death modeled as equivalent to SoC, a more favorable ICER estimate was achieved $(€ 7,903 / \mathrm{QALY}$ gained). Additionally, the results were insensitive to the consideration of titration visit cost and ECG cost in the model as well as to changes in utility estimates to other data source (SHIFT predicted versus external literature) or inclusion of an age-adjustment (higher utility loss as the modeled cohort aged).

\section{Subgroup analyses}

The ICER values have been estimated for a series of subgroup populations. These subgroup populations were designed with reference to the clinical trial protocol and previous SHIFT study populations. The subgroup analyses indicated that the use of ivabradine plus SoC seems to be more cost-effective alternative over SoC alone among patients with more severe heart failure (NYHA class III and IV), as the ICER becomes lower when the severity of heart failure increases. The results of subgroup analyses are presented in Table 5 . It should be noted that ivabradine plus SoC remains a cost effective alternative in all subgroups.

\section{Probabilistic sensitivity analysis}

In the base case analysis, probabilistic analysis confirms the deterministic results. In particular, the analysis showed that ivabradine plus SoC is the more cost-effective comparator (assuming a willingness to pay threshold of $€ 36,000)$ for the majority of the run. The probabilistic sensitivity analysis showed that the likelihood of Ivabradine plus SoC being cost-effective at a willingness-topay threshold of $€ 36,000 /$ QALY was found to be $95 \%$ compared with SoC alone. The CEAC confirms that a willingness-to-pay threshold of $€ 36,000$ ivabradine plus $\mathrm{SoC}$ was the regimen of choice (Figure 3 ).

\section{Discussion}

In the present study, a Markov modeling approach was used to evaluate the cost-effectiveness of ivabradine in a lifetime horizon for CHF patients with a baseline heart rate $\geq 75$ b.p.m., compared with current standard of care, in Greece. The analysis revealed that ivabradine plus SoC 


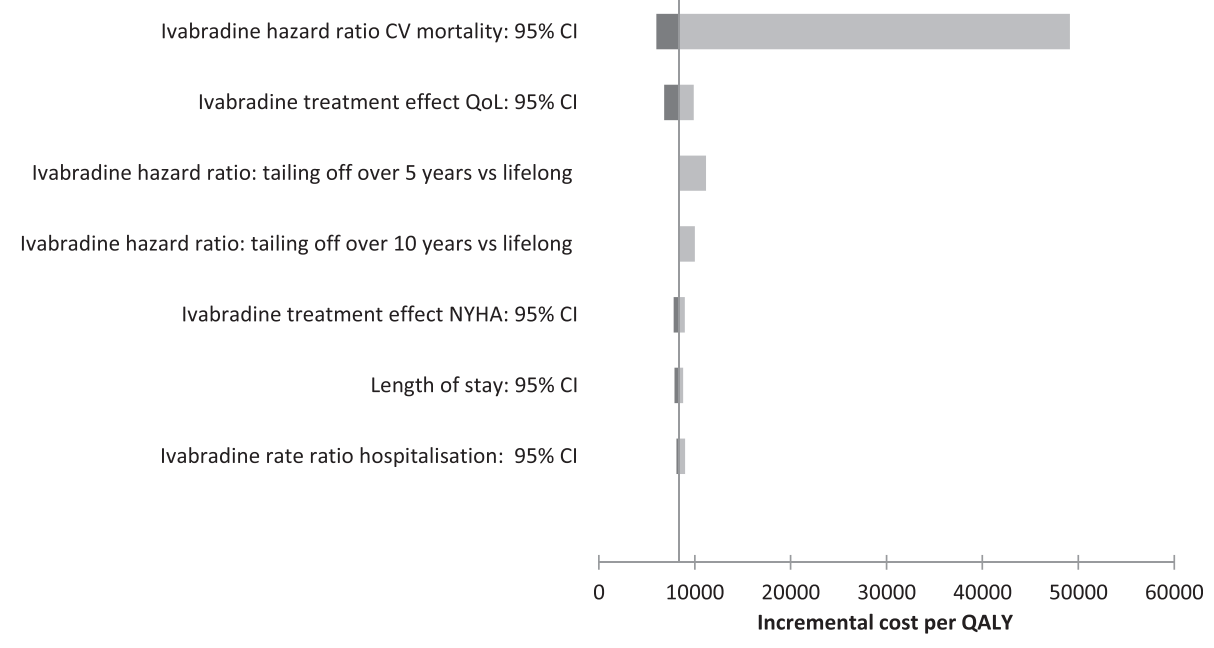

Figure $\mathbf{2}$ Tornado diagram: results from one way sensitivity analysis.

appeared to be more effective and more costly compared to SoC, with an ICER well below the threshold of $€ 36,000$ per QALY gained, indicating that ivabradine is a costeffective alternative for the management of CHF in Greece.

Our results are consistent with those submitted to NICE, in which ivabradine plus $\mathrm{SoC}$ was found to be associated with an increased cost of $£ 8,498$ per QALY gained compared with SoC. The ICER was sensitive to changes in the treatment effects of ivabradine at the upper bound 95\% confidence interval for CV mortality (HR: 0.80-1.03). However, the risk equations were developed using data from the total SHIFT population (heart rate $\geq 70 \mathrm{bpm}$ ). Therefore, this scenario analysis overestimates the upper bound hazard ratio and ICER estimate for the licensed indication (heart rate $\geq 75 \mathrm{bpm}$ ) [11].

Although the methodology adopted followed the standard recommendations and various sensitivity analyses were conducted to fully explore uncertainty, the analysis cannot substitute for real-life direct comparisons amongst the alternative treatments. Hence, post-launch observational studies are needed to verify the conclusions obtained from analyses such as the present. A more complete analysis from a broader (societal) perspective may also be worthwhile. True health care and patient direct and indirect costs are higher than those considered in the present analysis, and therefore the cost-effectiveness of a new therapy may be more favourable from a societal perspective. Moreover, in the present analysis it was assumed that the clinical outcomes obtained from the SHIFT trial were applicable to the Greek health care setting. The use of data derived from clinical trials may be questionable, however given the lack of locally generated data, this was the only relevant source for clinical data; one may argue that pivotal trials are almost universally used to build models for pricing and reimbursement decisions. Moreover,

Table 4 Summary of structural sensitivity analysis

\begin{tabular}{llll}
\hline Parameter & Min ICER & Max ICER & Output differences \\
\hline Treatment effect: HF only endpoints & $€ 7,903$ & $€ 9,196$ & $€ 1,293$ \\
CV: mortality Kaplan Meier vs Gompertz & $€ 9,196$ & $€ 10,313$ & $€ 1,117$ \\
Ivabradine treatment duration: 5 years vs lifelong & $€ 8,145$ & $€ 9,196$ & $€ 1,051$ \\
NYHA extrapolation: LoCF vs Assumption based & $€ 9,099$ & $€ 9,669$ & $€ 569$ \\
QoL weights: Exclude age adjustment vs include age adjustment & $€ 9,099$ & $€ 9,353$ & $€ 254$ \\
CV mortality: Weibull vs Gompertz & $€ 8,946$ & $€ 250$ \\
QoL weights: SHIFT predicted vs external literature & $€ 8,859$ & $€ 240$ \\
NYHA extrapolation: LoCF vs SHIFT predicted & $€ 9,070$ & $€ 9,099$ & $€ 133$ \\
CV mortality: exponential vs gompertz & $€ 9,077$ & $€ 9,196$ & $€ 126$ \\
Titration visit and ECG costs excluded vs included & $€ 9,099$ & $€ 22$
\end{tabular}


Table 5 Results from subgroup analyses

\begin{tabular}{|c|c|c|c|c|c|c|c|c|c|}
\hline \multirow[t]{2}{*}{ Subgroup } & \multicolumn{2}{|c|}{ Standard care } & \multicolumn{2}{|c|}{ Ivabradine + Standard care } & \multicolumn{5}{|c|}{ Incremental costs \& outcomes } \\
\hline & $\begin{array}{l}\text { Total costs } \\
\text { (in } € \text { ) }\end{array}$ & $\begin{array}{l}\text { Total } \\
\text { QALYs }\end{array}$ & $\begin{array}{l}\text { Total costs } \\
\text { (in } € \text { ) }\end{array}$ & $\begin{array}{l}\text { Total } \\
\text { QALYs }\end{array}$ & $\begin{array}{l}\text { Costs } \\
\text { (in } € \text { ) }\end{array}$ & LYs & QALYs & $\begin{array}{l}\text { Cost/LY } \\
\text { (in } € \text { ) }\end{array}$ & $\begin{array}{l}\text { Cost/QALY } \\
\text { (in } € \text { ) }\end{array}$ \\
\hline All patients ( $H R \geq 75$ b.p.m.) & 5,873 & 3.99 & 8,665 & 4.27 & 2,792 & 0.25 & 0.28 & 11,002 & 9,986 \\
\hline Age $<75$ years & 6,033 & 4.14 & 8,932 & 4.43 & 2,899 & 0.27 & 0.29 & 10,897 & 9,909 \\
\hline Age $\geq 75$ years & 4,350 & 2.54 & 6,123 & 2.69 & 1,772 & 0.14 & 0.16 & 12,944 & 11,355 \\
\hline NYHA ॥ & 6,358 & 4.55 & 9,498 & 4.84 & 3,140 & 0.24 & 0.28 & 12,935 & 11,112 \\
\hline NYHA III & 5,528 & 3.55 & 8,059 & 3.83 & 2,531 & 0.27 & 0.28 & 9,542 & 9,044 \\
\hline NYHA IV & 3,383 & 1.79 & 4,726 & 2.00 & 1,343 & 0.22 & 0.21 & 6,018 & 6,445 \\
\hline HF duration $<0.6$ years & 6,683 & 5.02 & 10,063 & 5.35 & 3,379 & 0.28 & 0.33 & 12,085 & 10,287 \\
\hline HF duration $\geq 0.6<2$ years & 5,881 & 4.10 & 8,752 & 4.39 & 2,871 & 0.26 & 0.29 & 10,919 & 9,883 \\
\hline HF duration $\geq 2<4.8$ years & 5,489 & 3.66 & 8,138 & 3.92 & 2,648 & 0.24 & 0.26 & 10,916 & 10,168 \\
\hline HF duration $\geq 4.8$ years & 5,466 & 3.20 & 7,755 & 3.44 & 2,290 & 0.23 & 0.24 & 9,931 & 9,527 \\
\hline No beta blocker & 5,144 & 3.08 & 7,384 & 3.39 & 2,240 & 0.31 & 0.31 & 7,239 & 7,267 \\
\hline Beta blocker $<$ half target dose & 5,481 & 3.60 & 7,992 & 3.87 & 2,511 & 0.25 & 0.27 & 9,942 & 9,250 \\
\hline Beta blocker $=>$ half target dose $<$ target dose & 6,338 & 4.45 & 9,438 & 4.73 & 3,100 & 0.24 & 0.28 & 12,794 & 11,115 \\
\hline Beta blocker $=>$ target dose & 6,440 & 4.64 & 9,684 & 4.92 & 3,244 & 0.24 & 0.28 & 13,602 & 11,619 \\
\hline LVEF $<26 \%$ & 5,407 & 3.31 & 7,709 & 3.60 & 2,302 & 0.28 & 0.29 & 8,356 & 8,072 \\
\hline LVEF $\geq 26 \%<30 \%$ & 5,483 & 3.66 & 8,052 & 3.94 & 2,569 & 0.25 & 0.27 & 10,163 & 9,440 \\
\hline LVEF $\geq 30<33 \%$ & 5,993 & 4.19 & 8,947 & 4.47 & 2,955 & 0.26 & 0.28 & 11,558 & 10,400 \\
\hline LVEF $\geq 33 \%$ & 6,443 & 4.64 & 9,688 & 4.92 & 3,245 & 0.23 & 0.27 & 13,977 & 11,807 \\
\hline Non-diabetic & 5,746 & 4.04 & 8,577 & 4.32 & 2,831 & 0.25 & 0.28 & 11,210 & 10,112 \\
\hline Diabetic & 6,150 & 3.86 & 8,859 & 4.14 & 2,709 & 0.26 & 0.28 & 10,556 & 9,710 \\
\hline No prior CAD & 5,839 & 4.20 & 8,719 & 4.52 & 2,880 & 0.29 & 0.32 & 9,907 & 9,051 \\
\hline Prior CAD & 5,887 & 3.90 & 8,643 & 4.16 & 2,756 & 0.24 & 0.26 & 11,549 & 10,448 \\
\hline
\end{tabular}

HR: Heart rate; CAD: coronary artery disease; HF: Heart Failure; LY: life Years; QALY: Quality-adjusted-life year; NYHA: New York Heart Association; LVEF: Left Ventricular Ejection Fraction.

only two local experts and not an expert panel was used to obtain local resource utilization and validate some of the assumptions considered in the model. This fact might raise concerns about the subjectivity of model inputs and leave space for challenging the study results. Nonetheless, he is a well-known cardiologist with extensive clinical experience on management of CHF, in Greece. Finally, it should be noted that the results have to be considered in the strict Greek setting and on the basis of the present time resource and drug prices. If any of the underlying parameters change, so may the results and the conclusions of the analysis.

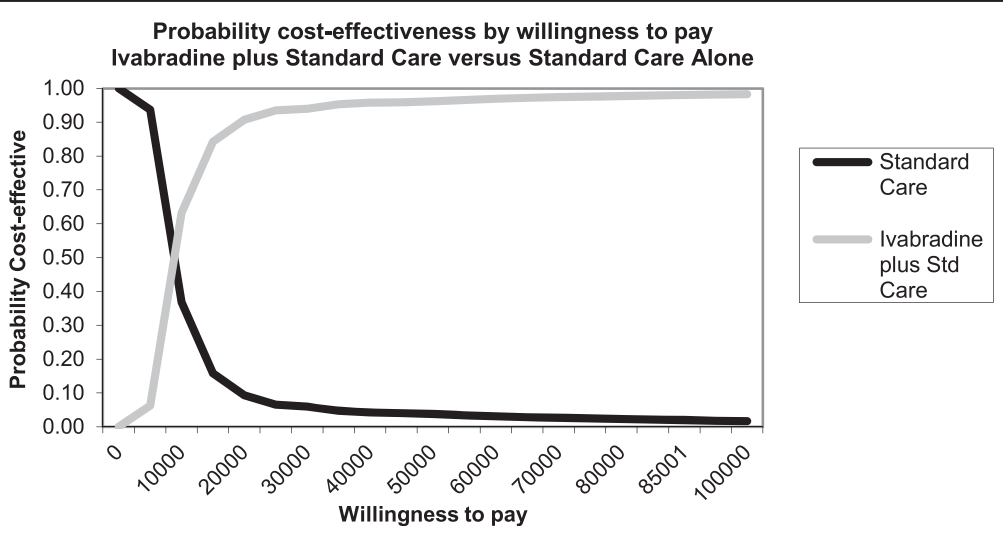

Figure 3 The acceptability curve for lifetime analysis. 


\section{Conclusions}

We can conclude that the use of Ivabradine plus SoC therapy may be regarded as a cost effective alternative when compared with SoCpy alone.

\section{Additional files}

\section{Additional file 1: Health care utilization and drug cost.}

Additional file 2: Proportion of patients using each standard therapy and mean drug daily dose.

\section{Abbreviations}

CHF: Chronic heart failure; SoC: Standard care; HF: Heart failure; NYHA: New York Heart Association; HR-QoL: health-related quality of life; QoL: Quality of life; ACE: angiotensin-converting-enzyme; ARBs: angiotensin receptors blockers; CV: Cardiovascular; HR: Hazard ratio; Cl: Confidence interval; NICE: National Institute for Health and Care Excellence; LOS: Length of stay; ICU: Intensive care unit; DRGs: Diagnostic related groups; GDP: Gross domestic product; QALY: Quality adjusted life year; LY: Life year; ICER: Incremental cost effectiveness ratio; CEACs: Cost-effectiveness acceptability curves; ECG: Electrocardiographic changes; LVEF: Left ventricular ejection fraction; LoCF: Last observation carried forward.

\section{Competing interests}

B.A. and BN is a Servier employee. However, the study sponsor had no interference in the study design, data collection or writing of the manuscript. None of the rest of authors has any personal or financial conflict of interest.

\section{Authors' contributions}

GK and CM adapted the model, conducted the analyses, interpreted the results and wrote the manuscript. NM supervised the study, contributed to results interpretation, and reviewed the manuscript. $A B$ and NB contributed to data collection and reviewed the manuscript. JP and AK were the medical experts provided local input data and reviewed the manuscript. All authors read and approved the final manuscript.

\section{Acknowledgments}

Authors would like to thank Servier-Hellas that sponsored this study.

\section{Author details}

${ }^{1}$ The Stavros Niarchos Foundation-Collaborative Center for Clinical Epidemiology and Outcomes Research (CLEO), National and Kapodistrian University of Athens, School of Medicine, Athens, Greece. ${ }^{2}$ Department of Cardiology, Attikon University Hospital, Athens, Greece. ${ }^{3}$ Department of Cardiology, Athens General Hospital "Georgios Genimmatas", Athens, Greece. ${ }^{4}$ Servier Hellas Pharmaceuticals Ltd, Athens, Greece. ${ }^{5}$ Department of Health Services Organization \& Management, National School of Public Health, Athens, Greece. ${ }^{6}$ Servier Laboratories Ltd, Suresnes, France.

Received: 23 April 2014 Accepted: 2 December 2014 Published online: 11 December 2014

\section{References}

1. Krum H, Stewart S: Chronic heart failure: time to recognize this major public health problem. Med J Australia 2006, 184:147-148.

2. Mosterd A, Hoes AW: Clinical epidemiology of heart failure. Heart 2007, 93:1137-1146

3. Cowie MR, Wood DA, Coats AJ, Thompson SG, Suresh V, Poole-Wilson PA, Sutton GC: Survival of patients with a new diagnosis of heart failure: a population based study. Heart 2000, 83:505-510.

4. The Criteria Committee of the New York Heart Association: Nomenclature and Criteria for Diagnosis of Diseases of the Heart and Great Vessels. 9th edition. Boston: Mass: Little, Brown \& Co; 1994.

5. McMurray JJ, Adamopoulos S, Anker SD, Auricchio A, Bohm M, Dickstein K, Falk V, Filippatos G, Fonseca C, Gomez-Sanchez MA, Jaarsma T, Kober L, Lip GY, Maggioni AP, Parkhomenko A, Pieske BM, Popescu BA, Ronnevik PK, Rutten FH, Schwitter J, Seferovic P, Stepinska J, Trindade PT, Voors AA, Zannad F, Zeiher A, Guidelines ESCCFP: ESC Guidelines for the diagnosis and treatment of acute and chronic heart failure 2012: The Task Force for the Diagnosis and Treatment of Acute and Chronic Heart Failure 2012 of the European Society of Cardiology. Developed in collaboration with the Heart Failure Association (HFA) of the ESC. Eur Heart J 2012, 33:1787-1847.

6. Liao L, Allen LA, Whellan DJ: Economic burden of heart failure in the elderly. Pharmacoeconomics 2008, 26:447-462.

7. Kaye DM, Krum H: Drug discovery for heart failure: a new era or the end of the pipeline? Nat Rev Drug Discov 2007, 6:127-139.

8. Ivabradine for chronic heart failure? Drug Ther Bull 2012, 50:117-120.

9. Swedberg K, Komajda M, Bohm M, Borer JS, Ford I, Dubost-Brama A, Lerebours G, Tavazzi L, Investigators S: Ivabradine and outcomes in chronic heart failure (SHIFT): a randomised placebo-controlled study. Lancet 2010, 376:875-885.

10. Briggs A, Claxton K, Sculpher M: Decision modelling for health economic evaluation. New York: Oxford University Press Inc; 2006.

11. Griffiths A, Paracha N, Davies A, Branscombe N, Cowie MR, Sculpher M: The cost effectiveness of ivabradine in the treatment of chronic heart failure from the UK National Health Service perspective. Heart 2014.

12. National Institute of Health and Care Excellence (NICE). Guide to the methods of technology appraisal 2013. Process and methods guides. [http://www.nice.org.uk/article/pmg9/resources/non-guidance-guide-to-themethods-of-technology-appraisal-2013-pdf]

13. Kind P, Hardman G, Macran S: UK Population norms for the EQ-5D. York Centre for Health Economics Discussion Paper 1999, 172.

14. Greek Ministry of Health.s Diagnostic Related Groups. [http://www.yyka. gov.gr/articles/health/domes-kai-draseis-gia-thn-ygeia/kwdikopoihseis/709kleista-enopoihmena-noshlia-1]

15. Greek Ministry of Health.s Drug price bulletin. [http://www.yyka.gov.gr/]

16. Government Gazzette: Presidential Decree 81/88, FEK 36/29-2-88 vol.A, "Costing of Medical Visits \& Operations". Athens, Greece; 1988.

17. Eichler HG, Kong SX, Gerth WC, Mavros P, Jonsson B: Use of cost-effectiveness analysis in health-care resource allocation decision-making: how are cost-effectiveness thresholds expected to emerge? Value Health: I Int Soc Pharm Outcomes Res 2004, 7:518-528.

18. Devlin N, Parkin D: Does NICE have a cost-effectiveness threshold and what other factors influence its decisions? A binary choice analysis. Health Econ 2004, 13:437-452.

19. World Health Organization.: WHO Commission on Macroeconomics and Health: Macroeconomics and Health: Investing in Health for Economic Development. Report of the Commission on Macroeconomics and Health. In Book WHO Commission on Macroeconomics and Health: Macroeconomics and Health: Investing in Health for Economic Development. Report of the Commission on Macroeconomics and Health. City; 2001.

20. WHO: Choosing interventions that are Cost Effective WHO-CHOICE, Cost-effectiveness thresholds. Available at http://www.who.int/choice/ costs/CER thresholds/en/index.html, 2013.

21. IMF, 2012. International Monetary Fund, World Economic Outlook Database, October 2012. Available at http://www.imf.org/external/pubs/ft/weo/2012/ 02/weodata/index.aspx

22. Briggs A, Claxton K, Sculpher M: Desicion modelling for health economic evaluation. Oxford: Oxford University Press; 2006.

23. Fenwick E, O'Brien BJ, Briggs A: Cost-effectiveness acceptability curves-facts, fallacies and frequently asked questions. Health Econ 2004, 13:405-415.

doi:10.1186/s12913-014-0631-0

Cite this article as: Kourlaba et al.: Economic evaluation of ivabradine in the treatment of chronic heart failure in Greece. BMC Health Services Research 2014 14:631. 